\title{
A PRACTICAL METHOD FOR DESIGN OF COASTAL STRUCTURES IN SHALLOW WATER
}

\author{
Henk Jan Verhagen ${ }^{1}$, Gerbrant van Vledder ${ }^{1,2}$, Sepehr Eslami Arab ${ }^{1}$ \\ Modern design formula for coastal structures (like rock stability formula and overtopping \\ formula use wave parameters $\left(H_{2 \%}\right.$ and $\left.T_{m-1,0}\right)$ which are not readily available from \\ standard boundary condition wave data. For transforming values like $H_{s}$ and $T_{p}$ to the \\ parameters used in the new formulas, often-fixed conversion factors are used. However, \\ this may lead to significant errors. Therefore, it is better to calculate these new parameters \\ with an appropriate wave transformation model. The one-dimensional Graphical User \\ Interface for SWAN (SwanOne) is presented as a simple tool to perform the required \\ transformation.
}

\section{INTRODUCTION}

Recent research has shown that for wave structure interaction in case of shallow water, the spectral period based on the first negative moment of the energy $\operatorname{spectrum}\left(T_{m-1.0}\right)$ is a better descriptor than a mean period or the peak period of the spectrum. On this basis in the Rock Manual [2007], several equations for run-up, overtopping and structural stability are presented. Also in the new EurOtop Overtopping Manual [2007], this parameter is used. The Rock Manual also indicates that for structural stability the parameter $H_{2 \%}$ is a better descriptor than the $H_{s}$ or the $H_{m 0}$. However, the determination of these parameters is not yet standard procedure, and often conversion values are used (e.g. $H_{2 \%} / H_{m 0}=1.4$ and $T_{m-1,0} / T_{p}=1.1$; and therefore implicitly assuming a Rayleigh distribution and a Jonswap spectral shape with peak enhancement factor $\gamma$ $=3.3$ and an $f^{5}$ spectral tail).

However, by using these standard conversion factors the advantages of the new approach completely disappear, because for non-standard coasts the conversion factors are different because the near shore spectral shape differs from deep water. Exactly in those cases, the new approach is valuable.

\section{NEW GUIDANCE}

\section{The Rock Manual}

The Rock Manual [2007] gives two sets of equations for the determination of the stability of rock armour. In the manual, the equations 5.136/137 are for deep water and the equations 5.139/140 are for shallow water. The validity of

\footnotetext{
${ }^{1}$ Delft University of Technology, Faculty of Civil Engineering and Geosciences, Section Hydraulic Engineering, PO Box 5048, NL2600GA Delft, The Netherlands,

H.J.Verhagen@tudelft.nl; G.P.VanVledder@tudelft.nl;S.EslamiArab@student.tudelft.nl

2 Alkyon, PO Box 248, NL8300AE Emmeloord, The Netherlands, Vledder@Alkyon.nl
} 
these formulas is in the manual indicated by means of a figure (Figure 1 in this paper). Each set consists of an equation for plunging waves and an equation for surging waves. In this paper only the plunging conditions will be discussed, since for surging waves an identical elaboration can be followed.

The definition of "shallow" water is not very exact. An intermediate area exists between the validity of the deep and shallow equations. Therefore it is handy to come to one single formula valid for both deep and shallow water conditions.

\begin{tabular}{|c|c|c|c|}
\hline \multirow[b]{2}{*}{ Item } & \multicolumn{3}{|c|}{ Water depth characterisation } \\
\hline & Very shallow water & Shallow water & Deep water \\
\hline $\begin{array}{l}\text { Parameter: } \\
\text { Relative water depth at the toe: } h / H_{\text {s.toe }}\end{array}$ & $\approx 1.5-\approx 2$ & $<3$ & $>3$ \\
\hline Wave height ratio, $R_{H}=H_{\text {s.toe }} / H_{\text {so }}$ & $<70 \%$ & $70 \%<R_{H}<90 \%$ & $>90 \%$ \\
\hline $\begin{array}{l}\text { Stability formulae: } \\
\text { Van der Meer - deep water, } \\
\text { Equation nos } 5.136 \text { and } 5.137\end{array}$ & & & \\
\hline $\begin{array}{l}\text { Van der Meer - shallow water } \\
\text { Equation nos } 5.139 \text { and } 5.140\end{array}$ & & & \\
\hline
\end{tabular}

Figure 1: Validity of the rock stability equations according to the Rock Manual [2007]

The formula for deep water presented in the Rock Manual is:

$$
\frac{H_{s}}{\Delta D_{n 50}}=c_{p l} P^{0.18}(S / \sqrt{N})^{0.2} 1 / \sqrt{\xi_{m}}
$$

in which $c_{p l}=6.2$ with a standard deviation of 0.4 (so for design one should apply $\left.c_{p l}=5.5\right)$.

For shallow water conditions, the Rock Manual recommends:

$$
\frac{H_{s}}{\Delta D_{n 50}}=c_{p l} P^{0.18}(\mathrm{~S} / \sqrt{N})^{0.2}\left(\frac{H_{s}}{H_{2 \%}}\right) 1 / \sqrt{\xi_{m-1,0}}
$$

In which $c_{p l}=8.4$ with a standard deviation of 0.7 (so for design apply $c_{p l}=$ 7.25).

In deep water, assuming a Rayleigh distribution there is a fixed relation between:

$$
\begin{aligned}
& \left.H_{S}=1.41 H_{2 \%} \quad \text { and (assuming a Jonswap spectrum with } \gamma=3.3\right) \\
& T_{m}=0.92 T_{m-1,0} \quad\left(T_{p}=1.20 T_{m} \text { and } T_{p}=1.1 T_{m-1,0}\right)
\end{aligned}
$$

This means that both equations 5.136 and 5.139 can be written as: 


$$
\frac{H_{2 \%}}{\Delta D_{n 50}}=c_{p l} P^{0.18}(S / \sqrt{N})^{0.2} 1 / \sqrt{\xi_{m-1,0, H_{2 \%}}}
$$

(for details is referred to the Appendix).

Note that in the Iribarren number also the parameter $H_{2 \%}$ is used instead of $H_{s}$. Of course, the coefficient $c_{p l}$ and $c_{s u}$ are different for plunging and surging waves:

- For shallow water $c_{p l}=8.4$ and $c_{s u}=1.3$ (direct from Rock Manual)

- For deep water $\quad c_{p l}=8.2$ and $c_{s u}=1.5$ (converted values)

This difference is small, but not negligible. The coefficients for equation 5.139 originate from Van Gent et.al [2003]. Because most of the tests in that paper are for shallow water, the Rock Manual recommends using the Van Gent coefficients for shallow water and the Van der Meer coefficients for deep water. Some probable reason for the difference between the coefficients has been discussed by Verhagen et al.[2006] as well as by Muttray and Reedijk [2008].

In conclusion one finds that for both deep and shallow water conditions calculations best can be carried out using the $H_{2 \%}$ and the $T_{m-1,0}$.

\section{The Overtopping Manual}

For the calculation of run-up and overtopping all available and recommendable methods are presented in the EurOtop manual [2007]. Nearly all equations in this manual use $T_{m-1,0}$ as a parameter for the wave period. For the wave height, in general the $H_{m 0}$ is used. However, the manual recommends, "Although prediction methods in this manual are mainly based on the spectral significant wave height, it might be useful in some cases to consider also other definitions, like the $2 \%$-wave height $H_{2 \%}$ or $H_{1 / 10}$, the average of the highest 1/10-the of the waves." Unfortunately, the manual does not recommend when to do this. In conclusion one finds that also for the calculation of run-up and overtopping the parameters the $H_{2 \%}$ and the $T_{m-1,0}$ are important.

\section{AVAILABLE TOOLS}

It is essential to determine the $H_{2 \%}$ and the $T_{m-1,0}$ as correct as possible. The use of "standard" conversion factors is not to be recommended, especially not in shallow water. This may lead to rather significant errors, as will be demonstrated in the example at the end of this paper.

\section{Determination of $\boldsymbol{H}_{2 \%}$}

For the determination of the $\mathrm{H}_{2 \%}$ the Battjes-Groenendijk method [Battjes and Groenendijk, 2000] can be applied. The method is quite straightforward, because the required parameters are all given in the mentioned paper. The method is based on data from a flume experiment with foreshore slopes of 1:20 to $1: 250$.

However, all these slopes are for continuous slopes, not a varying profile. According to Battjes and Groenendijk, the effect of the bottom slope is of 
secondary nature. In case of a varying bed slope, it is suggested to use the average local bed slope, measured over a wavelength offshore of the point under consideration.

\section{Determination of $\boldsymbol{T}_{\boldsymbol{m}-1,0}$}

In order to obtain a value for $T_{m-1,0}$, one needs the local shallow water wave spectrum. Such a spectrum can be obtained by flume tests or by using a spectral wave model, like sWAN. For daily engineering use, sWAN [Booij et.al, 1999] is used with a Graphical User Interface (GUI). For swAN, different organisations have produced GUI's which are commercially available. Most GUI's require as input a two dimensional depth matrix; but for many cases a one dimensional

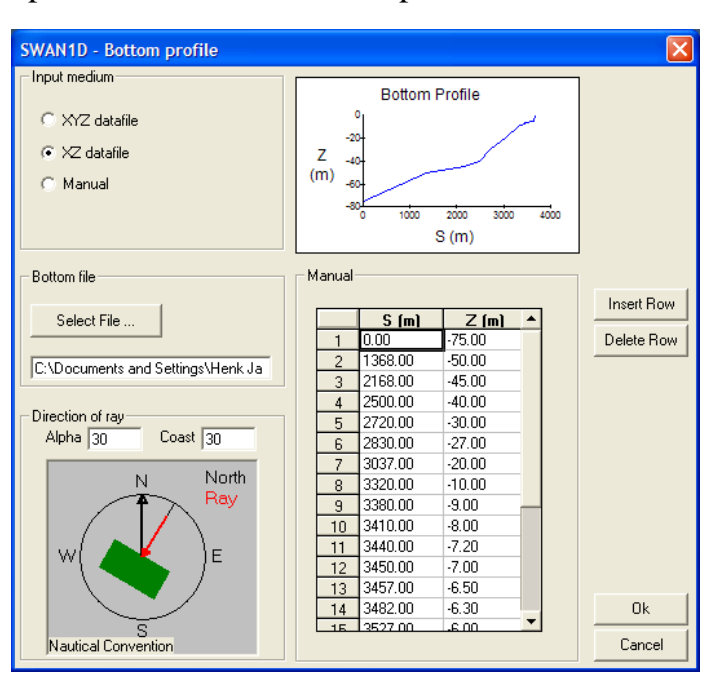
computation is sufficient. For simple calculations SWAN, even running via a user friendly GUI, is still quite some work.

Therefore, an existing onedimensional GUI for SWAN has been updated. This new GUI, SwanOne, requires as input only a simple (format free) ASCII file with one profile (distances and depths), as well as the deepwater wave height, mean wave period and direction.

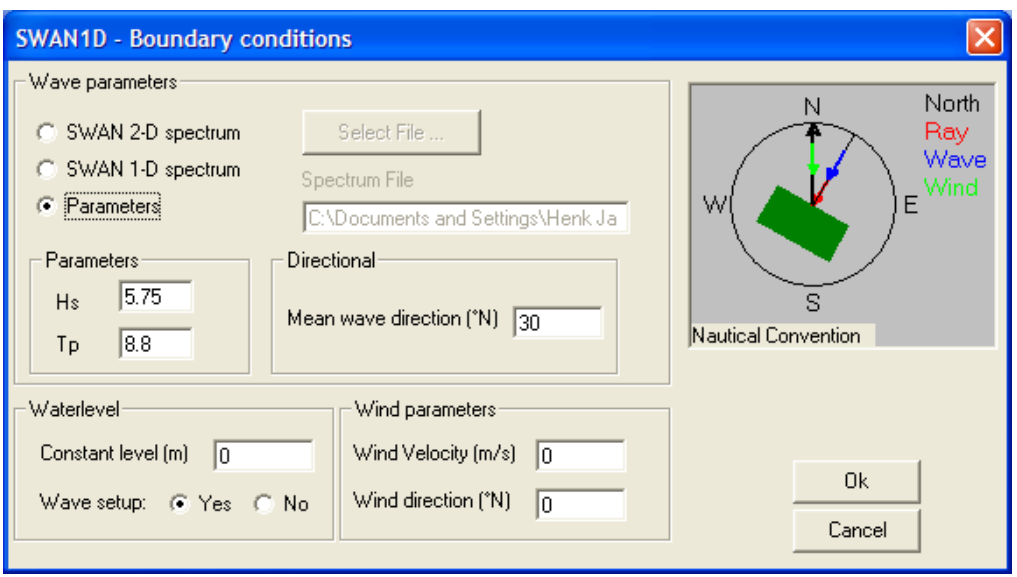

Figure 2: Typical input screens of SwanOne 
One may also enter the orientation of the profile, the direction of the waves, as well as additional wind and/or currents. The output of SwanOne is at any selected point not only the spectrum, but also values of $T_{m-1,0}, H_{m 0}$ and $H_{2 \%}$.

The previous version of SwanOne was written in Visual Basic and had an in-build version of swAN. The disadvantage of this approach was that new releases of swAN could not be implemented in SwanOne in an easy way. The new version of SwanOne is written as MatLab routines. It is available as a set of routines as well as in the form of a compiled version, which can be used without having a MatLab licence.

Although the package uses the one-dimensional option of SWAN, it is still possible to calculate directional spectra. Since the one-dimensionality only comprises the spatial variation, the only limitation is in fact that the package assumes parallel depth contour lines. For many engineering problems this is a very acceptable limitation.

Operation of SwanOne is very easy. An input-file has to be made containing the depth information. This is a simple ASCII-file with distance and depth (with respect to Chart Datum). In the menus, one can define the orientation of the coastline, the profile, the wave direction. A wind speed and wind direction can be added, as well as a waterlevel (difference with Chart Datum). In addition, optional current data can be entered. Wave heights at the seaward boundary are given as wave height $\left(H_{m 0}\right)$ and

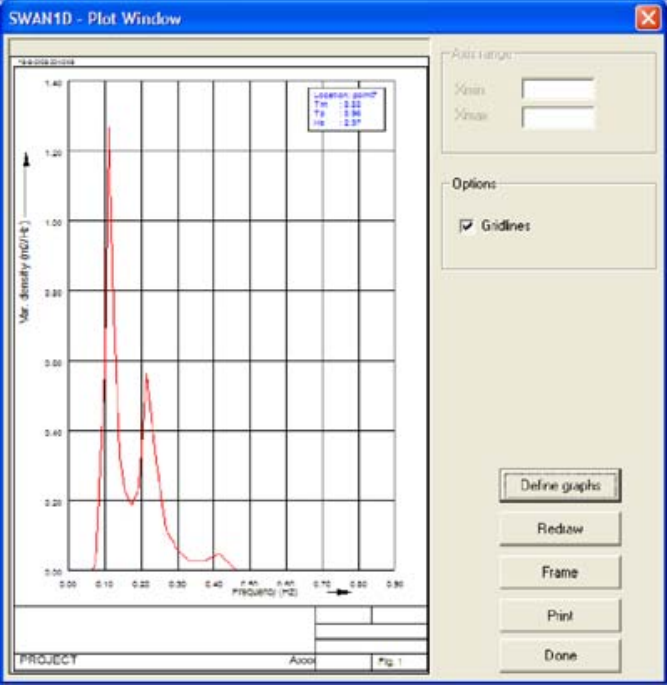

Figure 3: Sample output spectrum wave period $\left(T_{p}\right)$ and an incident wave direction $\theta$. The program then calculates the input spectrum (assuming a Jonswap spectrum). Alternatively, one may also enter a file with an arbitrary spectrum.

Two types of output are possible, a graph of key parameters as a function of the distance, and spectra on pre-selected locations. 


\section{CALCULATIONS PERFORMED}

In order to investigate if such a simplified one-dimensional approach is useful, a number of computations were made. For this example, a coastline is selected with steep deep-water slope and a wide, shallow shelf with a width of nearly $5 \mathrm{~km}$ (see Figure 4). Very near to the coast there is a steep slope leading to the waterline. We have a deep-water wave with $H_{s}=5.8 \mathrm{~m}$ and a $T_{p}=$ $9 \mathrm{~s}$, with a mean Jonswap spectrum $\quad(\gamma=3.3)$. The waves approach the coast under an angle of $30^{\circ}$. There is no wind and no current.

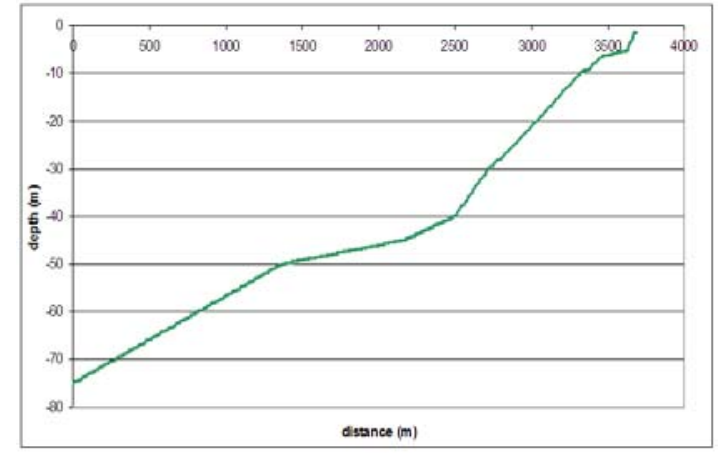

Figure 4. Demo profile
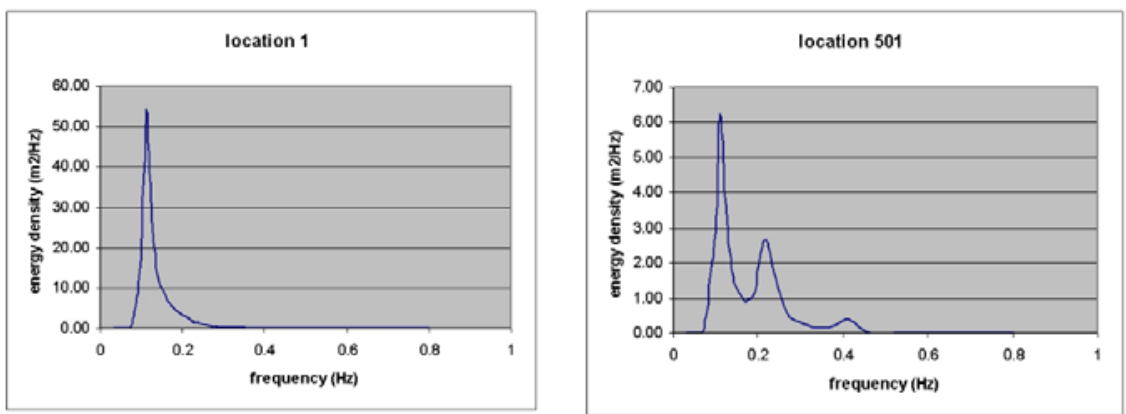

Figure 5: Spectrum in deep water and very near to the waterline

Figure 5 shows the transformation of the spectrum. This means that also the measure for the wave period changes. In Figure 6 the ratios between $T_{p}$ and $T_{m-1,0}$ as well as $T_{m}$ and $T_{m-1,0}$ are shown. It is clear that using a constant ratio (handbooks often give $T_{p}=1.1 T_{m-1,0}$ ) is not very correct. Besides, the ratio between $H_{m 0}$ and $H_{2} \%$ is not a constant value. This is indicated in Figure 7. The $\mathrm{H}_{2 \%}$ is computed with the Battjes-Groenendijk method.

\section{Note on wave steepness}

In many formulae the Iribarren number is used, being the ratio of the structure slope and the square root of the wave steepness. In fact, in most formulas it is not the intention to include the physical parameter "wave steepness", 
but the physical parameters "dimensionless ratio wave height over wave period".

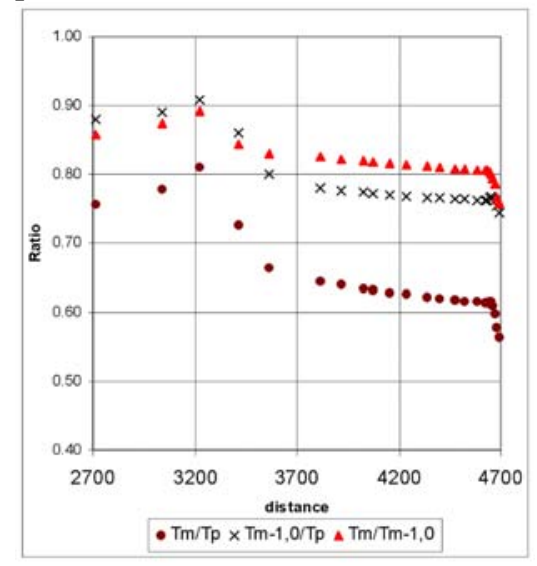

Figure 6: Ratio between $T_{m 0}$ and $T_{m-1,0}$

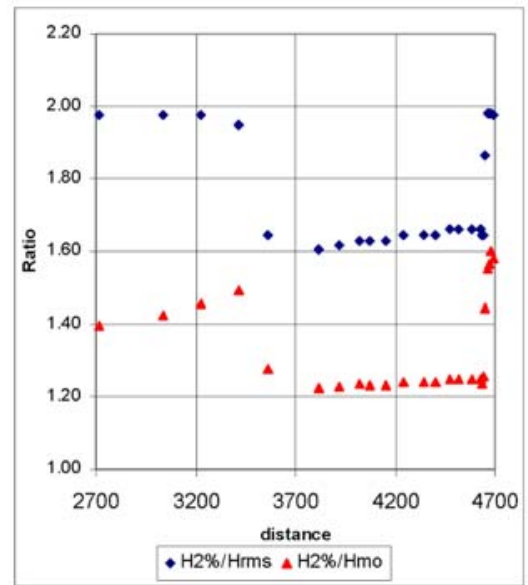

Figure 7: Ratio between $\boldsymbol{H}_{m 0}$ and $\boldsymbol{H}_{2 \%}$

A good way of making this parameter dimensionless is not using the wave period itself, but multiply the period with $g /(2 \pi)$. This is the "deep-water wave length", however, it should be calculated with the local wave period. Thus, in fact one is using a fictitious deep-water wave length.

$\tilde{L}=\frac{g}{2 \pi} T_{m-1,0 ; \text { local }}^{2}$

This may result in a fictitious wave steepness:

$S_{m-1,0 ; \text { local }}=\frac{H_{m 0}}{\tilde{L}}$

and

$\xi_{m-1,0 ; \text { local }}=\frac{\tan \alpha}{\sqrt{\frac{H_{m 0}}{\tilde{L}}}}$

One should realise that the steepness used in this Iribarren number is only a computational value, and has no physical meaning. Therefore, the value of this fictitious steepness might be much more that the physical maximum wave steepness. A fictitious steepness may be more than 5\%. Figure 8 shows this phenomenon. For further details is referred to Heineke and Verhagen [2007].

In Figure 8 lines with Lxx are calculated using the local (shallow water) wavelength; the other lines use the deep water wave length. Tm indicates that the period $T_{m 0}$ is used, Tm- 1 means that $T_{m-1,0}$ is used. 


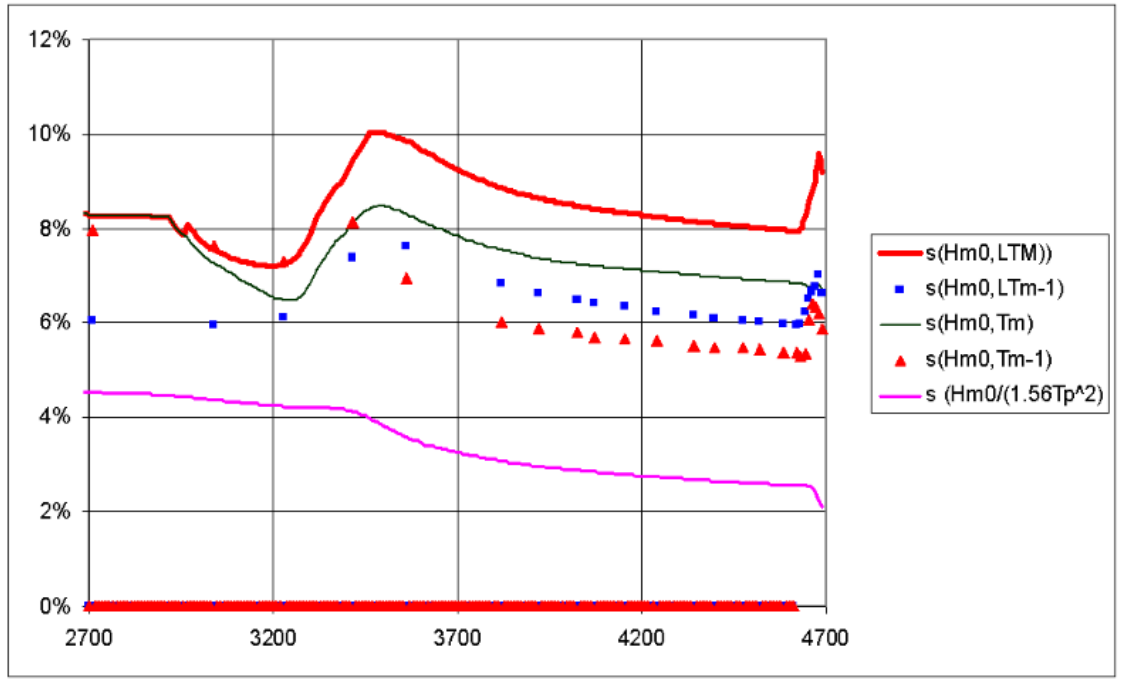

Figure 8: Various ways of defining the wave steepness

\section{Effect of the different parameters}

To compare the effect of the different parameters a calculation is made for the required rock size and the expected run-up for a construction build in front of the coast. The structure will be on the plateau at MSL -8 m.

Five different cases are calculated:

1. use $H_{2 \%}$ and $T_{m-1,0}$

2. use $H_{2 \%}$ and $T_{p} / 1.1$

3. use $1.4 H_{m 0}$ and $T_{m-1,0}$

4. use $1.4 H_{m 0}$ and $T_{p} / 1.1$

5. use local $H_{m 0}$ and $T_{m 0}$ and deep water formula for stability

This resulted in the following values:

\begin{tabular}{|l|l|l|l|l|l|}
\hline & 1 & 2 & 3 & 4 & 5 \\
\hline$D_{n 50}(\mathrm{~m})$ & 0.78 & 0.85 & 0.85 & 0.93 & 0.87 \\
\hline$W_{50}(\mathrm{~kg})$ & 1244 & 1606 & 1635 & 2127 & 1719 \\
\hline$R u(\mathrm{~m})$ & 8.4 & 10.0 & 9.2 & 10.7 & - \\
\hline
\end{tabular}

This sample calculation shows that the required stone size may vary between 1200 and $2200 \mathrm{~kg}$, depending on the choice of parameters. It stresses the importance of a correct choice of the parameters.

For armour layers often a class A grading according to European Standard EN13383 is required. This standard describes that for example for stones 1-3 tonnes $\left(\mathrm{HMA}_{1000 / 3000}\right.$ ) the W50 may only vary between 1700 and $2100 \mathrm{~kg}$. This range is considerably smaller than the range following from the differences in the various parameters. 


\section{THE SWANONE GRAPHICAL USER INTERFACE}

The SwanOne graphical user interface is written in MatLab. The program is available free in a compiled version. In order to run the compiled version the package MCR with the correct version has to be installed on your computer. This package can be installed using MCRinstaller, which is available with SwanOne. SwanOne uses the latest version of SWAN.

For users who do not want to download and install MCR on their computer (it is a quite large package), an older version of SwanOne is available. This package has similar features, but uses an older version of sWAN (40.01). The packages can be downloaded from:

- $\quad$ http://www.kennisbank-waterbouw.nl/Software or

- $\quad$ http://www.hydraulicengineering.tudelft.nl

For information on SWAN itself is referred to the official sWAN homepage:

- http://www.swan.tudelft.nl or

- $\quad$ http://fluidmechanics.tudelft.nl/swan

\section{CONCLUSIONS}

The new shallow water equations presented in the Rock Manual and the Overtopping Manual are only useful in case one is able to determine the shallow water boundary conditions with sufficiently high accuracy, and not only with conversion numbers. The tool SwanOne is able to perform these computations in a user friendly way. 


\section{APPENDIX}

Transformation of deepwater stability formula in terms of $\boldsymbol{H}_{2 \%}$ and $\boldsymbol{T}_{\boldsymbol{m}-1,0}$

Transformation constants:

$H_{2 \%}=1.4 H_{s}$ [Battjes and Groenendijk, 2000]

$T_{p}=1.1 T_{m-1,0}$ [Rock Manual, 2007]

$T_{p}=1.2 T_{m 01}$ [Goda 2000, table 2.4, $\gamma=3.3$ ]

Thus: $\quad T_{m}=0.92 T_{m-1,0}$

Steepness:

$$
\begin{gathered}
s_{f}=\frac{H_{s}}{L_{0}}=\frac{H_{2 \%}}{1.4} \frac{1}{\sqrt{1.56 T_{m}^{2}}}=\frac{H_{2 \%}}{1.4} \frac{1}{\sqrt{1.56 \cdot 0.92^{2} T_{m-1,0}^{2}}}= \\
=0.776 \frac{H_{2 \%}}{\sqrt{1.56 T_{m-1,0}^{2}}}=0.776 s_{m-1,0} \\
\xi_{m}=\frac{\tan \alpha}{\sqrt{s_{f}}}=\frac{\tan \alpha}{\sqrt{0.776 s_{m-1,0}}}=1.135 \xi_{m-1,0}
\end{gathered}
$$

Plunging wave:

$$
\begin{aligned}
\frac{H_{s}}{\Delta D_{n 50}} & =c_{p l}[\ldots .] \frac{1}{\sqrt{\xi_{m}}} \\
\frac{H_{2 \%}}{\Delta D_{n 50}} & =1.4 c_{p l}[\ldots .] \frac{1}{\sqrt{1.135} \sqrt{\xi_{m-1,0}}} \\
& =1.3145 c_{p l} \frac{1}{\sqrt{\xi_{m-1,0}}}
\end{aligned}
$$

Converted $c_{p l}$ coefficients for adapted equations:

- for calculation of average $1.3145 * 6.2=8.15$

- $\quad$ for design calculations $1.3145 * 5.5=7.23$

Surging wave:

$$
\frac{H_{s}}{\Delta D_{n 50}}=c_{s u}[\ldots .] \sqrt{\cot \alpha} \xi_{m}^{P}
$$




$$
\begin{aligned}
\frac{H_{2 \%}}{\Delta D_{n 50}} & =1.4 c_{s u}[\ldots .] \sqrt{\cot \alpha}\left[1.135 \xi_{m-1,0}\right]^{P} \\
& \left.=1.49 c_{s u}[\ldots .] \sqrt{\cot \alpha} \xi_{m-1,0}^{P} \quad \text { (assuming } P \approx 0.5\right)
\end{aligned}
$$

Converted $c_{s u}$ coefficients for adapted equations:

- for calculation of average $1.49 * 1.0=1.49$

- $\quad$ for design calculations $1.49 * 0.87=1.30$

\section{REFERENCES}

Battjes, J.A., Groenendijk, H.W. [2000] Wave height distribution on shallow foreshores. Coastal Engineering, Vol 40, No.3, pp.161-182.

Booij, N., Ris, R.C., Holthuijsen, L.H. [1999] A third generation wave model for costal regions, Part I: model description and validation. J. of Geophysical Research 104 (C4) 7649-7666.

Goda, Y. [2000] Random seas and design of maritime structures. World Scientific, Singapore.

Heineke, D., Verhagen, H.J. [2007] On the use of the fictitious wave steepness and related surf-similarity parameter in methods that describe the hydraulic and structural response to waves. Proc. $4^{\text {th }}$ Coastal Structures Conference, Venice, Italy.

Muttray, M., Reedijk, B. [2008] Reanalysis of breakwater stability with steep foreshores, proc.31 ${ }^{\text {st }}$ ICCE, Hamburg, Germany.

Overtopping Manual [2007] EurOtop; Wave overtopping of sea defences and related structures - Assessment manual. EA-Environmental Agency, UK; ENW-Expertise Netwerk Waterkeren, NL; KFKI-Kuratorium für Forschung im Küsteningenieurwesen, DE. See www.overtoppingmanual.com.

Rock Manual [2007] CIRIA, CUR, CETMEF. The Rock Manual. The use of rock in hydraulic engineering (2nd edition). C683, CIRIA, London.

Van der Meer, J.W. [1988] Rock slopes and gravel beaches under wave attack, $\mathrm{PhD}$ thesis, Delft University of Technology, Delft.

Van Gent, M.R.A., Smale, A.J., Kuiper, C. [2003] Stability of rock slopes with shallow foreshores. Proc. $3^{\text {rd }}$ Coastal Structures Conference, Portland, USA.

Verhagen, H.J., Reedijk, J., Muttray, M. [2006] The effect of foreshore slope on breakwater stability. Proc. $30^{\text {th }}$ ICCE, San Diego, USA. 